\title{
Energy Harvester Based on the Synchronization Phenomenon of a Circular Cylinder
}

\author{
Junlei Wang, ${ }^{1,2}$ Jingyu Ran,, and Zhien Zhang ${ }^{1,2}$ \\ ${ }^{1}$ Key Laboratory of Low-Grade Energy Utilization Technologies and Systems, Chongqing University, Ministry of Education, \\ Chongqing 400030, China \\ ${ }^{2}$ School of Power Engineering, Chongqing University, Chongqing 400030, China
}

Correspondence should be addressed to Jingyu Ran; ranjy@cqu.edu.cn

Received 18 February 2014; Revised 4 April 2014; Accepted 6 April 2014; Published 12 May 2014

Academic Editor: Shen Yin

Copyright (C) 2014 Junlei Wang et al. This is an open access article distributed under the Creative Commons Attribution License, which permits unrestricted use, distribution, and reproduction in any medium, provided the original work is properly cited.

\begin{abstract}
A concept of generating power from a circular cylinder undergoing vortex-induced vibration (VIV) was investigated. Two lead zirconate titanate (PZT) beams which had high power density were installed on the cylinder. A theoretical model has been presented to describe the electromechanical coupling of the open-circuit voltage output and the vibration amplitudes based on a secondorder nonlinear Van der pol equation and Gauss law. A numerical computation was applied to measure the capacity of the power generating system. The lift and drag coefficient and the vortex shedding frequency were obtained to verify how the nondimensional parameter reduced velocity $U_{r}$ affects the fluid field. Meanwhile, a single-degree of freedom system has been added to describe the VIV, presynchronization, and synchronization together with postsynchronization regimes of oscillating frequencies. And the amplitudes of the vibration have been obtained. Finally, the vibrational amplitudes and the voltage output could go up to a high level in the synchronization region. The maximum value of the voltage output and the corresponding reduced velocity $U_{r}$ were $8.42 \mathrm{~V}$ and 5.6, respectively.
\end{abstract}

\section{Introduction}

Energy harvesting from ambient environment has been proposed for powering electronic components. Many published literatures were focused on the microsized power generator harvesting energy from vibration, temperature difference, and rotating or some others [1-5]. Some traditional ways like batteries that have a finite life span and require expensive and hard maintenance cannot be employed in many places. Nowadays a great effort has been made to the development of wireless sensor works (WSN) [6-9], which can be used in many fields like environmental protection, military monitoring, and structural health. Energy harvesting from environment can produce an enduring and battery-free power source for the WSN. However, the power harvester using vortex energy in small scale millimeter and micron is not commonly reported as many as others. Vortex power is a kind of nonpollution energy source ever presented in the natural environment. Converting the flow power into useful electrical power is very important for the WSN and other autonomous systems in various applications.

Flow behaviour around a cylindrical obstacle has become the subject of much discussion and measurement [10], and vortex-induced vibration (VIV) is a kind of self-excited vibration [11]. Here a nondimensional parameter reduced velocity $U_{r}=U / f_{c} D$ (where $f_{c}$ is the natural frequency $(\mathrm{Hz}), D$ is the feature size of the bluff body $(\mathrm{m})$, and $U$ is the coming flow velocity $(\mathrm{m} / \mathrm{s})$ ) has been used to indicate the intensity of flow field. When $U_{r}$ has come to a suitable value, which means the vortex shedding frequency is under a similar value of the natural frequency of vibrational system, then a lock-in or synchronization phenomenon will happen. VIV is experienced in many situations; for instance, VIV on the cables of bridges, electric wires, high buildings, pipes, towing 
cables and mooring lines [12] subjected to vortex, and water currents may suffer fatigue damage. Thus the reduction of VIV has become a major topic in design engineering [1318]. However, due to the fact that the concepts of focusing on collecting power by VIV are proposed in recent decades, it has to be taken into account in the design of microelectronics devices and electrical equipment like sensors requiring for sustaining power supply [6-8]. As a development of models focusing on crosswise VIV which follow the idea of a wake oscillator model [19-22], a variable model of the fluctuating nature of vortex shedding was created to describe the wake dynamics and it was conceived to satisfy a Van der Pol or Rayleigh equation modelling a self-sustained oscillation of finite amplitude [11]. Thus, a second-order nonlinear equation has to be solved to get the vibrational amplitude to catch the energy of the vortex shedding.

VIV under different Reynolds numbers (Re) has been overviewed by Williamson and Govardhan [10, 23]. For systems at low Reynolds numbers of a few hundreds, the characteristics of the laminar physics in VIV of circular cylinder and long elastic bodies of aerodynamically bluff cross-section (noncircular) have been studied by Pakinson, Naudascher, and Rockwell [24-26]. What is more, for some cases at high Reynolds numbers like $100000<\mathrm{Re}<400000$, characteristic of the severe turbulence has been discussed by Macdonald and Larose $[27,28]$ in recent years.

At the same time, there are mainly two piezoelectric materials employed in the vortex-induced vibration harvesters: polyvinylidene difluoride (PVDF) and lead zirconate titanate (PZT). PVDF film is usually used in vortex-induced vibration [29-31] because of its large elastic compliance whilst its poor piezoelectric performance and large impedance lead to a low energy harvesting efficiency in energy harvesting. At Cornell, Allen and Smits [29] worked on the behaviour of long and very flexible piezoelectric strips (called "eels") in the wake of a flat plate perpendicular to a flow. They presented the results of the structural response of the eels based on modal analysis and provided particle image velocimetry (PIV) images to support their predictions. A conclusion was drawn that for the maximum coupling between the eel and the vortex street, small stiffness, a matching of the eel's natural frequency alone with the vortex shedding frequency, is required. Akaydin et al. [31] explored aspects of energy harvesting from turbulent fluid flow using piezoelectric generators. It was found that matching the fluid flow's predominant frequency with the natural frequency of the piezoelectric generator appears to maximise the piezoelectric output voltage.

For harvesting energy from flow induced vibration (FIV), Akaydin et al. [32] designed an experiment of a piezoelectric energy transducer in the uniform flow field. Both experimental and computational results have been gotten and compared to estimate the aeroelectromechanical efficiency and level of harvested power. Abdelkefi et al. [33] and Mehmood et al. [34], investigated the possibility of harvesting energy from elastically-mounted circular cylinders. They used, respectively, a wake oscillator model and direct numerical simulations to determine the fluctuating lift force. Abdelkefi et al. [33] reported that the aerodynamic nonlinearity results in the presence of hardening behavior.
Both research studies determined the effects of the load resistance on the synchronization region and level of the harvested power. They demonstrated that maximum level of the harvested power can be associated with minimum values in the transverse displacement due to the shunt damping effect.

To investigate the possibility of harvesting energy from other flow-induced vibrations, different investigations have been performed [35-40]. Abdelkefi and Nuhait [35] investigated the effects of cambered wing-based piezoaeroelastic energy harvesters on the flutter speed and the performance of the harvester. Abdelkefi and his coauthors [36, 37] investigated the potential of harvesting energy from a combination of base excitations and VIV or galloping, respectively.

In Marine Renewable Energy Labortory (MRELab) at the University of Michigan, Bernitsas et al. [41] and Lee and Bernitsas [42] have developed the VIVACE (vortexinduced vibration for aquatic clean energy) to utilize the VIV phenomenon to generate power. To solve the VIV problem of a single cylinder with PTC, Wu et al. [43] has developed a CFD code in OpenFOAM software. Ding et al. [44] has developed a CFD code in OpenFOAM to solve the VIV of multiple circular cylinders.

Compared with PVDF, PZT has a good piezoelectric performance. The piezoelectric strain coefficient $d_{31}$ of PZT is 10 times larger than PVDF, while the flexibility coefficient is 40 times smaller than PVDF. Correspondingly, there appears to be a technological problem of how to make PZT cantilevers vibrate in environmental wind or water flow. Several researches have been conducted to put a rigid PZT cantilever beam behind the bluff body [45-47]. For instance, Gao et al. [46] have designed an energy harvester by putting a piezoelectric beam behind a cylinder. However, the PZT cantilever beam plays a role as a splitter plate, which can delay and eventually prevent the vortex-induced vibration (VIV). Hobbs [47] fixed a PZT transducer at the root and employed a cylinder as a sail to enhance the transducer vibration by vortex shedding in wind, with only $20 \mu \mathrm{w}$ output power. Previous works have been carried out to harvest energy by the principle of rectangular flexible beam selfexciting to amplify PZT cantilever vibration and generate power [29-34]. While a few researches focus on the lock-in or synchronization phenomenon which can largely enhance the vibrational amplitudes, and for the purpose of energy harvesting, it is significant to generate energy at low flow velocities for a better adaptation to sensors or actuators.

Thus, a great deal of work has been done in this paper by making use of the synchronization phenomenon in the vortex-induced vibration of circular cylinders under the lowvelocities $(94 \leq \operatorname{Re} \leq 150,0.06 \mathrm{~m} / \mathrm{s} \leq U \leq 0.094 \mathrm{~m} / \mathrm{s})$ free stream to harvest energy. In the present work, fluid flow over a circular cylinder is studied under low Reynolds number range of $94 \leq \operatorname{Re} \leq 150$ and an electromechanical coupling is achieved. In Section 2, the fluid flow and the piezoaeroelastic model and the coupling scheme are discussed. In Section 3, the validation of the fluid flow solver and the coupling scheme with previous experimental data as well as numerical results are presented. In Section 4, the effects of Reynolds number 


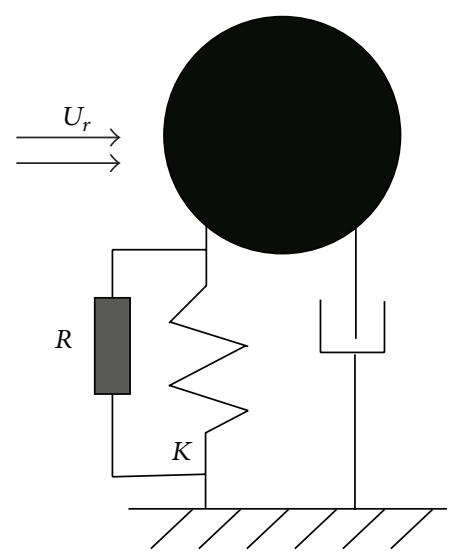

(a)

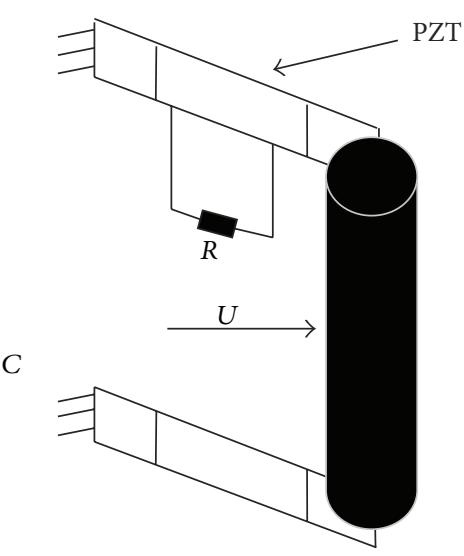

(b)

FIGURE 1: A schematic of the proposed cylinder-based piezoaeroelastic energy harvester.

on the vibrational amplitudes and electromechanical voltage output are demonstrated. Then in Section 5 the conclusions are given to summarise the work presented in this paper.

\section{The Present Approach}

We consider a VIV-based piezoaeroelastic energy harvester consisting of an elastically mounted circular cylinder and piezoelectric transducer attached to its transverse degree of freedom, as shown in Figures 1(a) and 1(b).

When this system is subjected to an incoming flow, the cylinder undergoes vortex-induced vibration in the transverse direction. If the vortex speed exceeds a critical value, the vibrational frequency will be locked near the natural frequency of the structure that is so-called the "lock-in" or "synchronism" phenomenon. From the performance analysis for inertial harvesters [48], the voltage output $(V)$ and the harvested power $(P)$ are proportional to vibration amplitude and the cube of the operation frequency

$$
\begin{aligned}
& P=\frac{V^{2}}{R}, \\
& P \propto y_{t},
\end{aligned}
$$

where $y_{t}$ is related to the tip displacement of the circular cylinder and $R$ is the electrical load resistance; in present approach, only the "lock-in" frequency is considered, and the computation and verification of the "lock-in" will be shown below.

2.1. The Fluid Solver. The key of solving the VIV of a circular cylinder is the fluid-structure interaction. In this work, a parallel CFD code is used. The incompressible continuity and unsteady Navier-Stokes equations are directly coupled with the cylinder motion. These mutual interactions can be described by the governing equations of the vibrating and flow system. For fluid flow, if flow speeds are less than 0.3 times of the speed of the sound, the flow can be considered incompressible. The incompressible flow of a Newtonian fluid such as air and water can be demonstrated by continuity and N-S (Navier-Stokes) equations as follows:

$$
\begin{gathered}
\frac{D \rho}{D t}+\rho S_{k k}=0, \\
\rho \frac{D V_{i}}{D t}=-\frac{\partial p}{\partial x_{i}}+\frac{\partial \tau_{i j}}{\partial x_{j}}, \\
\rho \frac{D}{D t}=-\frac{\partial}{\partial t}+V_{k} \frac{\partial}{\partial x_{k}},
\end{gathered}
$$

where $p$ is the fluid pressure, $\rho$ is the fluid density, $V_{i}$ is the vector of velocity, and $\tau_{i j}$ is the stress tensor. The equations are based on the nonslip wall boundary conditions on the surface of the moving bluff body. The displacement of the bluff body modifies the flow field through the boundaries. Equations (3) and (4) are given under the no slip boundary conditions on the surface of the moving structure:

$$
V(x, y)=V_{\Pi}(x, y),
$$

where $x, y \in \Pi$. $\Pi$ is the wet surface of the circular cylinder and $V_{\Pi}$ is the velocity of the moving cylinder; the variable quantity $p_{\Pi}$ acting on $\Pi$ can generate the forcing term $F_{y}$ in the structural equation as shown below; the piezoelectric transducer to the transverse degree of freedom is attached, meaning that the effects of $F_{x}$ are not available.

2.2. The Structural Solver. As depicted in Figure 1(a), the kinematic and electromechanical equation of the singledegree of freedom (SDOF) system can be given by Akaydin et al. [31-33]; a second-order nonlinear Van der pol equation and Gauss law are coupled as follows:

$$
\begin{gathered}
m \ddot{y}_{t}+c \dot{y}_{t}+k y_{t}-\theta v=F_{y}, \\
\theta \dot{y}_{t}+C_{p} \dot{v}+\frac{v}{R}=0,
\end{gathered}
$$


TABLE 1: Properties of the electromechanical system.

\begin{tabular}{llc}
\hline Symbol & Property & Value \\
\hline$m$ & Mass of the cylinder per & $0.2979 \mathrm{~kg}$ \\
$k$ & unit length & $579 \mathrm{~N} / \mathrm{m}$ \\
$c$ & Stiffness per unit length & $120 \mathrm{Nm} / \mathrm{s}$ \\
$D$ & Damping per unit length & $1.6 \mathrm{~mm}$ \\
$\xi$ & Diameter of the cylinder & 0.0012 \\
$f_{n}$ & Damping ratio & $7.016 \mathrm{~Hz}$ \\
$C_{p}$ & Natural frequency & $120 \mathrm{nF}$ \\
$\theta$ & Capacitance & $1.55 e^{-3} \mathrm{~N} / \mathrm{V}$ \\
\hline
\end{tabular}

where $m$ is the mass of the oscillation cylinder per unit, $c$ is the structural damping, and $k$ represents the stiffness. $F_{y}$ characterizes the time-dependent excitation of the fluid flow applied on the circular cylinder, $\theta$ is the electromechanical coupling term, $R$ is the electrical load resistance, $v$ is the voltage across this load resistance, and $C_{p}$ is the capacitance of the piezoelectric layer. The geometric and material properties are the same as Mehmood et al. [35] shown in Table 1 which is used to verify the solver.

In open-circuit analysis, the load resistance is set as $R=$ $\infty$, then the relation between the displacement $y_{t}$ and the open-circuit voltage $V_{\text {oc }}$ can be obtained:

$$
y_{t}=\frac{-C_{p}}{\theta} V_{\mathrm{oc}} .
$$

Substituting $V_{\mathrm{oc}}$ in (8) for (6), the open-circuit aeropiezoelectric coupling equation can also be given as

$$
m \ddot{y}_{t}+c \dot{y}_{t}+\left(k+\frac{\theta^{2}}{C_{p}}\right) y_{t}=F_{y} .
$$

Thus (9) can be considered a new type of second-order nonhomogeneous equation given by a Van der pol formation. Substituting the constant in Table 1 for (5) we obtain

$$
\begin{gathered}
a_{1} \ddot{y}_{t}+a_{2} \dot{y}_{t}+a_{3} y_{t}=F_{y}, \\
v_{\mathrm{oc}}=b y_{t},
\end{gathered}
$$

where $a_{1}=0.2979, a_{2}=0.0325, a_{3}=599.2$, and $b=$ 12916.7. Equations (10)-(11) are the minimum form to solve the electromechanical coupling in present work.

\section{Validation of the Solver of Fluid-Structure Interaction}

The fluid-structure interaction solver is verified by comparing the circular cylinder's displacement with experimental results of Bearman [49] and other numerical results by Mehmood et al. [35], Yang et al. [50] and Schulz and Kallinderis [51]. The global range of computation is $25 \times 25 \mathrm{D}$. The Reynolds number is increased from 94 to 150 with small increments. Correspondingly, the nondimensional velocity $U_{r}=U / f_{c} D$ is increased from 5.34 to 8.49 . We also note that the cylinder

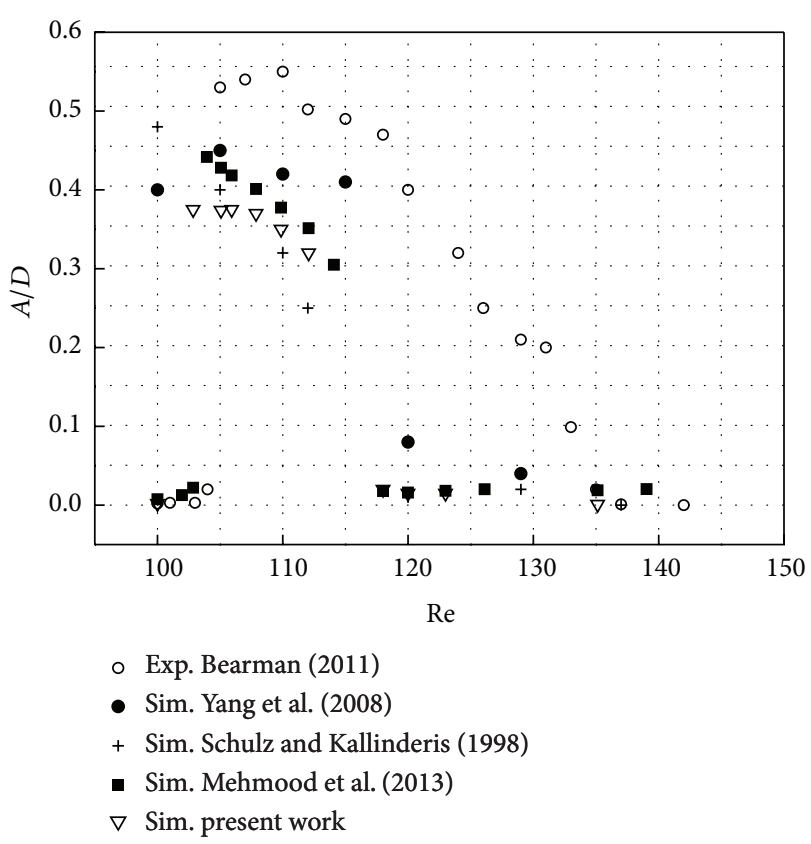

FIgURE 2: Comparison of maximum displacement amplitudes in present work $(\nabla)$ with the experimental measurements of Bearman [49] (-o) and numerical computations of Yang et al. [50] (•) and Schulz et al. [51] (+) and Mehmood et al. [35].

moves only in the transverse direction under the action of the flow force. The settings allow the cylinder to vibrate in the region of "lock-in" or "synchronization."

Figure 2 represents the nondimensional maximum oscillation amplitude $A / D$ of the cylinder as a function of the Reynolds number. From the pattern, we note that an obvious location of the bifurcation point or called onset of synchronization is captained and agrees well with the experimental results of Bearman [49]. Still, there are some small differences between our simulation and the previous simulations $[35,50,51]$ when comparing the maximum value of amplitude and the range of "lock-in". This can be due to the three-dimensional effects.

\section{The Effect of Reynolds Number on the System Power Outputs}

To estimate harvesting energy from the considered piezoelectric coupling system, we perform numerical simulation of the fluid flow around a rigid cylinder that moves in the transverse direction and has a piezoelectric beam attached on it. The goal of the present study is to power the sensors of small size. We limit the flow fluid to a Reynolds number in the range of $94 \leq \mathrm{Re} \leq 150$ that corresponds to a circular cylinder having a diameter of $1.6 \mathrm{~mm}$ and placed in a water stream of $0.06 \mathrm{~m} / \mathrm{s} \leq U \leq 0.094 \mathrm{~m} / \mathrm{s}$. The corresponding reduced velocity is in the range of $5.34 \leq U_{r} \leq 8.49$.

Time histories of the cylinder displacement, lift coefficient, and open-circuit voltage output are shown in Figures 


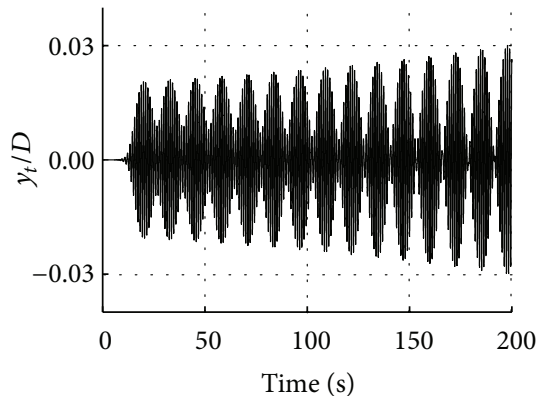

(a)

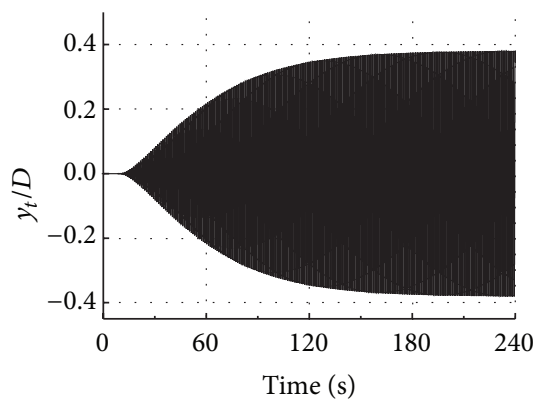

(d)

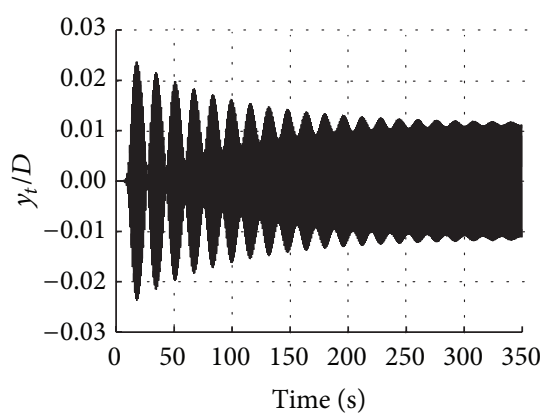

(g)

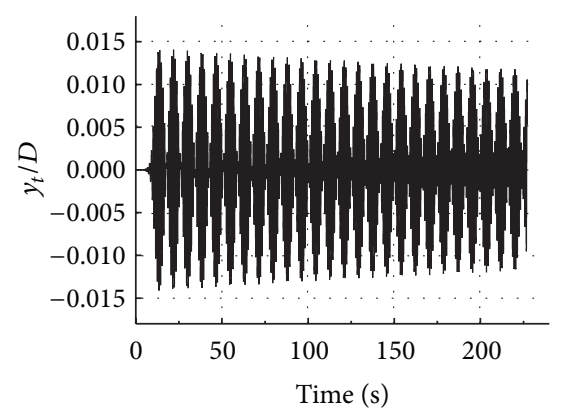

(j)

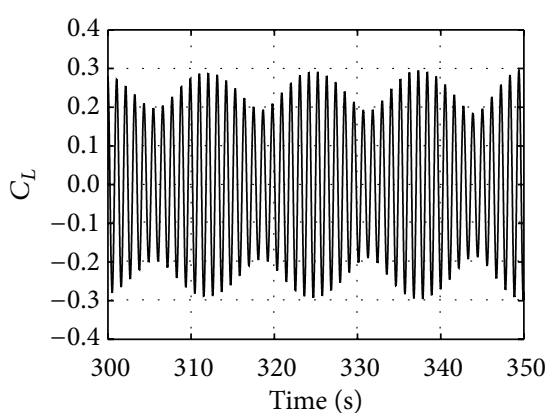

(b)

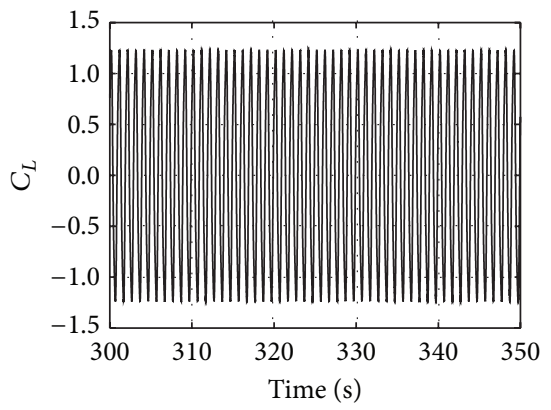

(e)

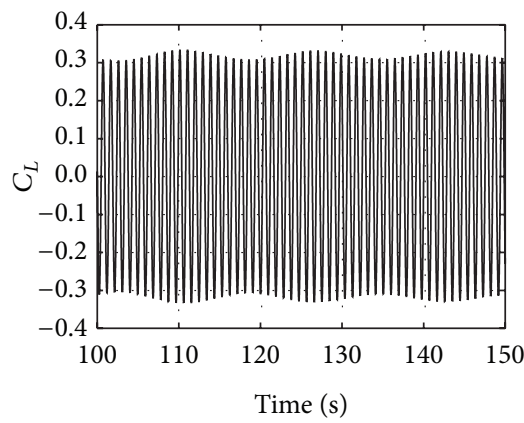

(h)

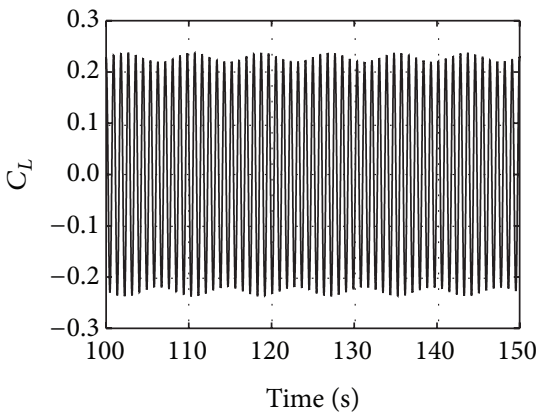

(k)

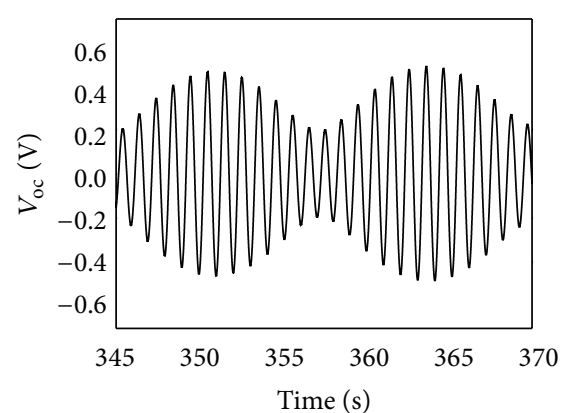

(c)

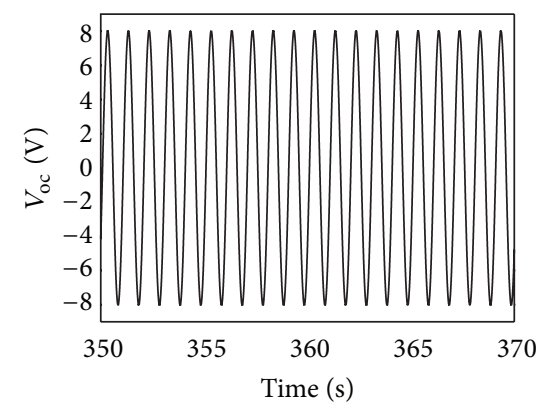

(f)

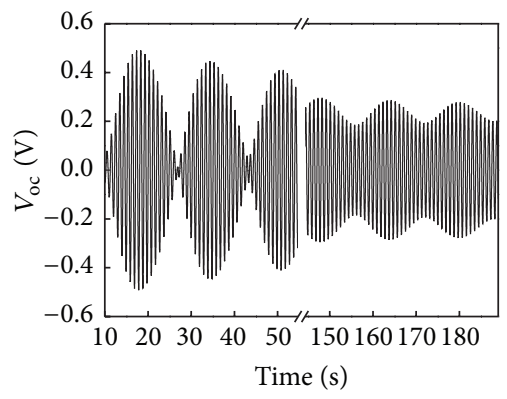

(i)

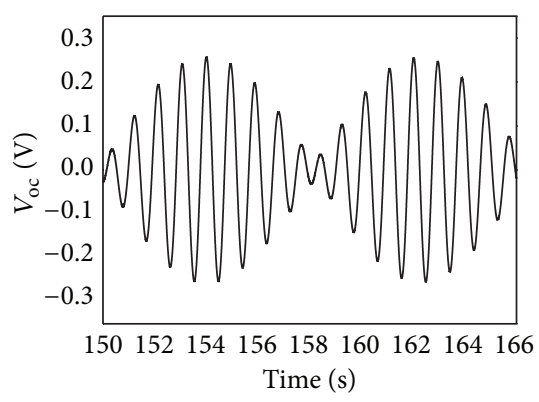

(l)

Figure 3: Time histories of the oscillating displacement, fluctuating lift coefficient, and open-circuit voltage output for the regions of presynchronous $(\operatorname{Re}=94)((a),(b)$, and $(\mathrm{c}))$, synchronous $(\operatorname{Re}=100)((d),(e)$, and $(\mathrm{f}))$, and postsynchronous $(\operatorname{Re}=109,113)((\mathrm{g}),(\mathrm{h})$, (i), (j), (k), and (l)).

3(a)-3(1), and three different regimes are given as presynchronous, synchronous, and postsynchronous. In presynchronous region $(\operatorname{Re}=94)$, the vibration of the cylinder remains very weak $\left(Y / D \sim 10^{-2}\right)$ and is enhanced gradually, as shown in Figure 3(a), and the reason is that the cylinder is in the transition state to the synchronous region. In Figure 3(b), the lift coefficient oscillates around a zero mean with a peak value of 0.28 . The response in this regime is 


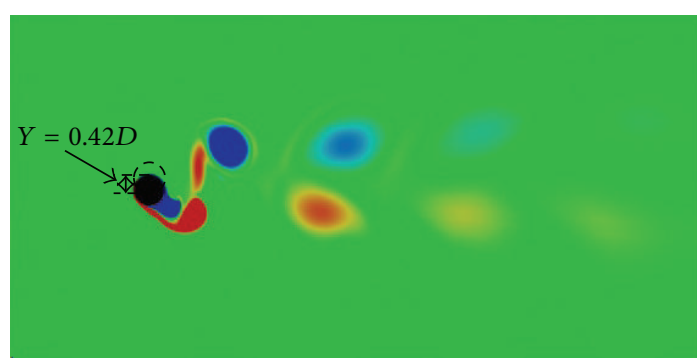

(a)

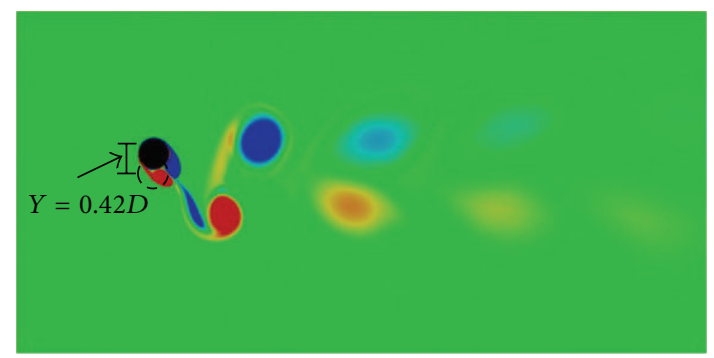

(b)

Figure 4: The vorticity contours of the two peak value times: (a) the cylinder moves to the bottom position in the flow and (b) the cylinder moves to the top position in the flow. Note that these two contours represent the synchronization region.

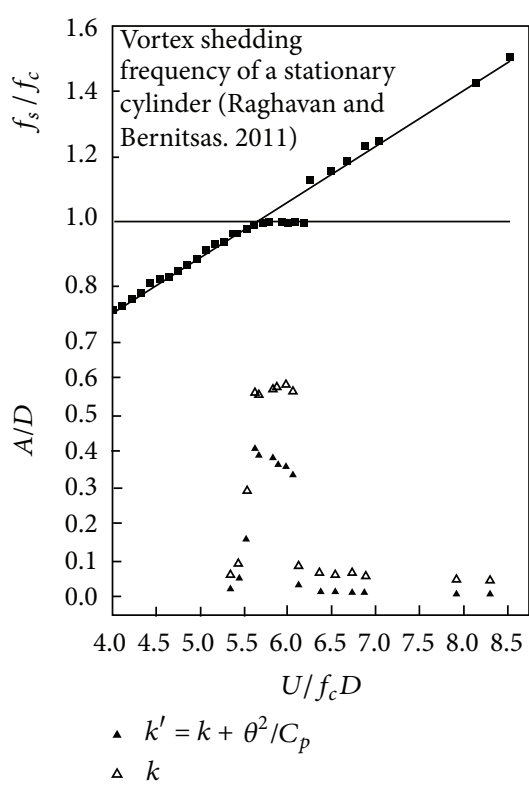

(a)

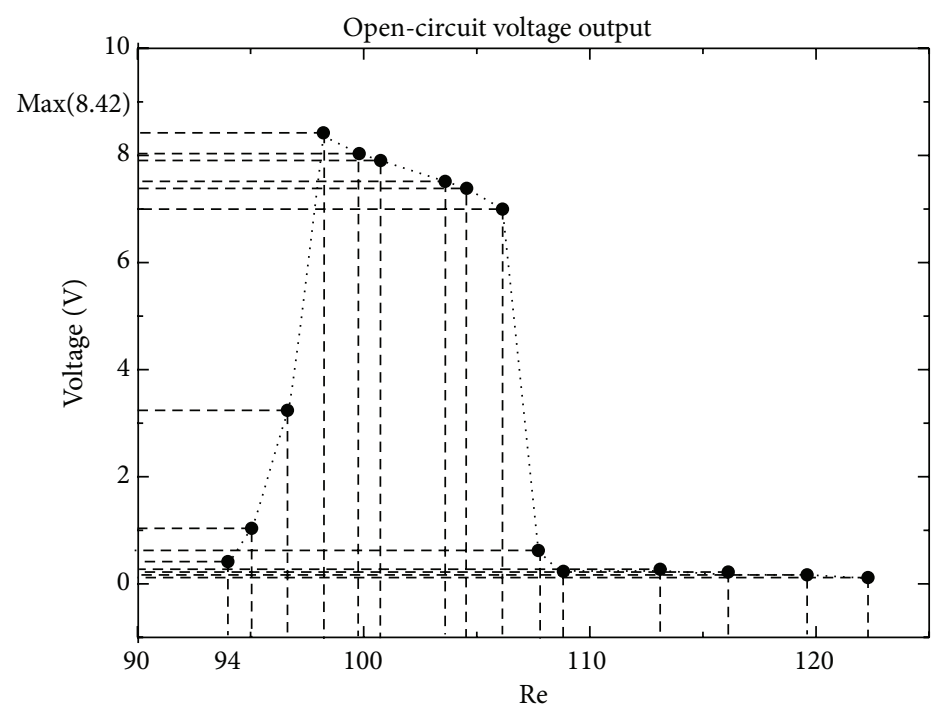

(b)

FIGURE 5: Synchronization region of (a) frequency and oscillating amplitude and (b) open-circuit voltage output of the circular cylinder.

quasi-periodic composing of two major frequencies, vortex shedding frequency and the natural frequency of the cylinder. The open-circuit voltage output in Figure 3(c) shows that its time history follows quite closely the oscillating displacement. Similar values for the displacement, lift coefficient, and voltage are obtained for Reynolds number up to 100 . At $\operatorname{Re}=100$, as shown in Figure 3(d), the amplitude of the oscillation reaches a synchronization region quickly and gets steady in a value of $Y / D=0.42$. Figures $4(\mathrm{a})$ and $4(\mathrm{~b})$ show the vorticity contours at this instance; two different locations of the cylinder are identified by the offset of $Y=$ $\pm 0.42 D$ to its initial position. A “ $2 S$ " mode vortex street is clearly shown in the region behind the circular cylinder. The lift and voltage output in Figures 3(e) and 3(f) also show periodic responses with a dominant frequency, that is, the cylinder natural frequency. The large vibration amplitudes extend up to $U=0.0687 \mathrm{~m} / \mathrm{s}$. The lift coefficient gets up to a value of $C_{L}=1.2$ and the open-circuit voltage output reaches $8.42 \mathrm{~V}$ high. This is due to the fact that the range of $100 \leq \operatorname{Re} \leq 109$ corresponds to the synchronization regime. From $\operatorname{Re}=109$, a bifurcates phenomenon of synchronization occurs. Oscillation amplitude, lift, and opencircuit voltage output shown in Figures 3(g), 3(h), and 3(j) go into another transition region close to that opposite to $\mathrm{Re}=$ 94. Again, the oscillation of the cylinder consists of two major frequencies. It is worth noting that the dominant frequency in $\operatorname{Re}=109$ is the cylinder natural frequency. However, at $\operatorname{Re}=94$, the dominant frequency is the vortex shedding frequency. In Figures 3(j), 3(k), and 3(l), it can be confirmed that the oscillation is in a continuous attenuation. For all the considered cases, we note that the time histories of the oscillation displacement and the output voltage have a same trend. Thus, we can conclude that the generated voltage is directly related to the displacement of the oscillating cylinder through (10).

Further insight into the different aspects of the oscillating displacement and frequency of different reduced velocities $U_{r}=U / f_{c} D$ is considered, as shown in Figure 5(a). The 
straight line plotted on the top of the figure represents the experimental results of the Strouhal number $S_{t}=f_{s} D / U$ by Raghavan and Bernitsas [52]. From the comparison of the nondimensional frequency $f=f_{s} / f_{c}$ with the straight line, the synchronization phenomenon can be clearly obtained from $5.6 \leq U_{r} \leq 6.2$. The corresponding computational results of the nondimensional oscillating amplitudes $A / D$ also show that the synchronization occurs in the same region of $U_{r}$. From Figure 5(b), a conclusion can be given that the high level voltage output can be achieved in the synchronization region. In this work, the max value of voltage output is $V_{\max }=8.42 \mathrm{~V}$ at $U_{r}=5.6$ or $\operatorname{Re}=100$. This means that for the optimum flow velocities, the maximum power generated from the VIV can be harvested.

\section{Conclusions}

The concept of energy harvesting from the vortex-induced vibration of a circular cylinder was investigated. The fluidsolid coupling of motion of the oscillating cylinder, piezoelectric transducer, simultaneously, and the flow field was solved using a server-class computer. The presynchronization, synchronization, and postsynchronization regimes were covered in the simulations performed in this work. Finally, the electromechanical coupling was achieved by the deduced formulas. The harvester response of open-circuit voltage output was analyzed. The discussions above lead to the following conclusions:

(1) a new concept to calculate harvested energy from the vortex-induced vibration is presented by deduced formulas in this paper. These formulas can be used directly to estimate open-circuit voltage output in an electromechanical VIV system;

(2) in the computational $\mathrm{Re}$ region, three different branches of VIV are obtained under different reduced velocities ranging from 5.34 to 8.49 . In the presynchronization regime, the dominant frequency of the oscillation is the vortex shedding frequency, while the dominant frequency of the oscillation is the natural frequency in postsynchronization regime;

(3) at $\operatorname{Re}=100$, the oscillation of the cylinder circular has come to a synchronization regime. The corresponding reduced velocity is $U_{r}=5.6$, and the amplitude of the oscillation increases rapidly to a peak value of $A / D=$ 0.42 . At $\operatorname{Re}=109$, the synchronization regime shifts from a transition region to the postsynchronization regime;

(4) in the synchronization regime, the voltage output of the VIV system is considerable which has a peak value of $8.42 \mathrm{~V}$;

(5) based on the screening criteria of maximum net power output, $U_{r}=5.6$ is optimum for the energy harvesting in the current model.

\section{Conflict of Interests}

The authors declare that there is no conflict of interests regarding the publication of this paper.

\section{Acknowledgment}

The authors gratefully acknowledge the financial support of this work by the Doctoral Program Found Project of the Ministry of Education of China (no. 20120191130003).

\section{References}

[1] S. P. Beeby, M. J. Tudor, and N. M. White, "Energy harvesting vibration sources for microsystems applications," Measurement Science and Technology, vol. 17, no. 12, article R175, 2006.

[2] N. S. Shenck and J. A. Paradiso, "Energy scavenging with shoemounted piezoelectrics," IEEE Micro, vol. 21, no. 3, pp. 30-42, 2001.

[3] A. Abdelkefi and M. Ghommem, "Piezoelectric energy harvesting from morphing wing motions for micro air vehicles," Theoretical \& Applied Mechanics Letters, vol. 3, Article ID 052001, 2013.

[4] K. A. Cook-Chennault, N. Thambi, and A. M. Sastry, "Powering MEMS portable devices-a review of non-regenerative and regenerative power supply systems with special emphasis on piezoelectric energy harvesting systems," Smart Materials and Structures, vol. 17, no. 4, Article ID 043001, 2008.

[5] A. Abdelkefi, A. Alothman, and M. R. Hajj, "Performance analysis and validation of thermoelectric energy harvesters," Smart Materials and Structures, vol. 22, no. 9, Article ID 095014, 2013.

[6] J. A. Paradiso and T. Starner, "Energy scavenging for mobile and wireless electronics," IEEE Pervasive Computing, vol. 4, no. 1, pp. $18-27,2005$.

[7] S. Roundy, P. K. Wright, and J. Rabaey, "A study of low level vibrations as a power source for wireless sensor nodes," Computer Communications, vol. 26, no. 11, pp. 1131-1144, 2003.

[8] G. K. Ottman, H. F. Hofmann, A. C. Bhatt et al., "Adaptive piezoelectric energy harvesting circuit for wireless remote power supply," IEEE Transactions on Power Electronics, vol. 17, no. 5, pp. 669-676, 2002.

[9] H.-K. Chang, D. Kim, and J. Park, "Design and analysis of portable loadless wind power source for ubiquitous sensor network," in Proceedings of the 2nd International Conference on Computer and Automation Engineering (ICCAE '10), pp. 93-96, February 2010.

[10] C. H. K. Williamson and R. Govardhan, "Vortex-induced vibrations," Annual Review of Fluid Mechanics, vol. 36, pp. 413455, 2004.

[11] W. Ding, Self-Excited Vibration, Springer, Berlin, Germany, 2011.

[12] S. O. Hansen, Vortex-Induced Vibrations of Structures, Structural Engineers World Congress, Bangalore, India, 2007.

[13] A. V. Belver, A. L. Ibán, and C. E. Lavín Martín, "Coupling between structural and fluid dynamic problems applied to vortex shedding in a $90 \mathrm{~m}$ steel chimney," Journal of Wind Engineering and Industrial Aerodynamics, vol. 100, no. 1, pp. 3037, 2012.

[14] Y. Itoh and T. Tamura, "The role of separated shear layers in unstable oscillations of a rectangular cylinder around a 
resonant velocity," Journal of Wind Engineering and Industrial Aerodynamics, vol. 90, no. 4, pp. 377-394, 2002.

[15] H. Ruscheweyh and G. Sedlacek, "Crosswind vibrations of steel stacks.-critical comparison between some recently proposed codes," Journal of Wind Engineering and Industrial Aerodynamics, vol. 30, no. 1, pp. 173-183, 1988.

[16] S. Schmidt and G. Solari, "3-D wind-induced effects on bridges during balanced cantilever erection stages," Wind and Structures, vol. 6, no. 1, pp. 1-22, 2003.

[17] R. Jankowski, M. Kujawa, and C. Szymczak, "Reduction of steel chimney vibrations with a pendulum damper," Task Quarterly, vol. 8, no. 1, pp. 71-78, 2004.

[18] A. Larsen, "A generalized model for assessment of vortexinduced vibrations of flexible structures," Journal of Wind Engineering and Industrial Aerodynamics, vol. 57, no. 2, pp. 281294, 1995.

[19] Y. Tamura and G. Matsui, "Wake-oscillator model of vortexinduced oscillation of circular cylinder," in Proceedings of the 5th International Conference on Wind Engineering, pp. 1085-1094, Fort Collins, Colo, USA, 1979.

[20] L. Mathelin and E. de Langre, "Vortex-induced vibrations and waves under shear flow with a wake oscillator model," European Journal of Mechanics B. Fluids, vol. 24, no. 4, pp. 478-490, 2005.

[21] A. Farshidianfar and H. Zanganeh, "A modified wake oscillator model for vortex-induced vibration of circular cylinders for a wide range of mass-damping ratio," Journal of Fluids and Structures, vol. 26, no. 3, pp. 430-441, 2010.

[22] W. Chen, L. Zhang, and M. Li, "Prediction of vortex-induced vibration of flexible riser using an improved wake-oscillator model," in Proceedings of the 28th International Conference on Ocean, Offshore and Arctic Engineering, vol. 5, Honolulu, Hawaii, USA, 2009.

[23] C. H. K. Williamson, "Vortex dynamics in the cylinder wake," Annual Review of Fluid Mechanics, vol. 28, no. 1, pp. 477-539, 1996.

[24] G. V. Parkinson, "Mathematical models of flow-induced vibrations of bluff bodies," in Flow-Induced Structural Vibrations, pp. 81-127, Springer, 1974.

[25] R. D. Blevins, Flow-Induced Vibration, D. Van Nostrand Reinhold Company, New York, NY, NY, 1990.

[26] E. Naudascher and D. Rockwell, Flow-Induced Vibrations, A. Balkema, Rotterdam, The Netherlands, 1994.

[27] J. H. G. Macdonald and G. L. Larose, "A unified approach to aerodynamic damping and drag/lift instabilities, and its application to dry inclined cable galloping," Journal of Fluids and Structures, vol. 22, no. 2, pp. 229-252, 2006.

[28] J. H. G. Macdonald and G. L. Larose, “Two-degree-of-freedom inclined cable galloping-part 1: general formulation and solution for perfectly tuned system," Journal of Wind Engineering and Industrial Aerodynamics, vol. 96, no. 3, pp. 291-307, 2008.

[29] J. J. Allen and A. J. Smits, "Energy harvesting eel," Journal of Fluids and Structures, vol. 15, no. 3, pp. 629-640, 2001.

[30] V. H. Schmidt, "Piezoelectric energy conversion in windmills," IEEE Ultrasonics Symposium Proceedings, pp. 897-904, 1992.

[31] H. D. Akaydın, N. Elvin, and Y. Andreopoulos, "Wake of a cylinder: a paradigm for energy harvesting with piezoelectric materials," Experiments in Fluids, vol. 49, no. 1, pp. 291-304, 2010.

[32] H. D. Akaydin, N. Elvin, and Y. Andreopoulos, "The performance of a self-excited fluidic energy harvester," Smart Materials and Structures, vol. 21, no. 2, Article ID 025007, 2012.
[33] A. Abdelkefi, M. R. Hajj, and A. H. Nayfeh, "Phenomena and modeling of piezoelectric energy harvesting from freely oscillating cylinders," Nonlinear Dynamics, vol. 70, no. 2, pp. 1377-1388, 2012.

[34] A. Abdelkefi and A. O. Nuhait, "Modeling and performance analysis of cambered wing-based piezoaeroelastic energy harvesters," Smart Materials and Structures, vol. 22, no. 9, Article ID 095029, 2013.

[35] A. Mehmood, A. Abdelkefi, M. R. Hajj, A. H. Nayfeh, I. Akhtar, and A. O. Nuhait, "Piezoelectric energy harvesting from vortexinduced vibrations of circular cylinder," Journal of Sound and Vibration, vol. 332, no. 19, pp. 4656-4667, 2013.

[36] H. L. Dai, A. Abdelkefi, and L. Wang, "Piezoelectric energy harvesting from concurrent vortex-induced vibrations and base excitations," Nonlinear Dynamics, 2014.

[37] Z. Yan and A. Abdelkefi, "Nonlinear characterization of concurrent energy harvesting from galloping and base excitations," Nonlinear Dynamics, 2014.

[38] A. Abdelkefi, Global nonlinear analysis of piezoelectric energy harvesting from ambient and aeroelastic vibrations [Ph.D. thesis], Virginia Tech, 2012.

[39] L. Zhao, L. Tang, and Y. Yang, "Comparison of modeling methods and parametric study for a piezoelectric wind energy harvester," Smart Materials and Structures, vol. 22, Article ID 125003, 2013.

[40] A. Abdelkefi, A. H. Nayfeh, and M. R. Hajj, "Modeling and analysis of piezoaeroelastic energy harvesters," Nonlinear Dynamics, vol. 67, no. 2, pp. 925-939, 2012.

[41] M. M. Bernitsas, K. Raghavan, Y. Ben-Simon, and E. M. H. Garcia, "VIVACE (Vortex Induced Vibration Aquatic Clean Energy): a new concept in generation of clean and renewable energy from fluid flow," Journal of Offshore Mechanics and Arctic Engineering, vol. 130, no. 4, Article ID 041101, 2008.

[42] J. H. Lee and M. M. Bernitsas, "High-damping, high-Reynolds VIV tests for energy harnessing using the VIVACE converter," Ocean Engineering, vol. 38, no. 16, pp. 1697-1712, 2011.

[43] W. Wu, M. M. Bernitsas, and K. Maki, "RANS simulation vs. experiments offlowinduced motion of circular cylinder with passive turbulence control at 35,000 $=\mathrm{Re}=130,000$," in Proceedings of the 30th International Conference on Ocean, Offshore and Arctic Engineering, pp. 733-744, ASME, Rotterdam, The Netherlands, June 2011.

[44] L. Ding, M. M. Bernitsas, and E. S. Kim, "2-D URANS vs. experiments of flow induced motions of two circular cylinders in tandem with passive turbulence control for $30,000<\operatorname{Re}<105,000$," Ocean Engineering, vol. 72, pp. 429-440, 2013.

[45] A. Khaligh, P. Zeng, and C. Zheng, "Kinetic energy harvesting using piezoelectric and electromagnetic technologies-state of the art," IEEE Transactions on Industrial Electronics, vol. 57, no. 3, pp. 850-860, 2010.

[46] X. Gao, W. H. Shih, and W. Shih, "Flow energy harvesting using piezoelectric cantilevers with cylindrical extension," IEEE Transactions on Industrial Electronics, vol. 60, no. 3, pp. 1116$1118,2013$.

[47] W. B. Hobbs, Piezoelectric energy harvesting: vortex induced vibrations in plants, soap films, and arrays of cylinders [Ph.D. thesis], Georgia Institute of Technology, 2010.

[48] P. D. Mitcheson, E. M. Yeatman, G. K. Rao et al., "Energy harvesting from human and machine motion for wireless electronic devices," Proceedings of the IEEE, vol. 96, no. 9, pp. 1457-1486, 2008. 
[49] P. W. Bearman, "Circular cylinder wakes and vortex-induced vibrations," Journal of Fluids and Structures, vol. 27, no. 5, pp. 648-658, 2011.

[50] J. Yang, S. Preidikman, and E. Balaras, "A strongly coupled, embedded-boundary method for fluid-structure interactions of elastically mounted rigid bodies," Journal of Fluids and Structures, vol. 24, no. 2, pp. 167-182, 2008.

[51] K. W. Schulz and Y. Kallinderis, "Unsteady flow structure interaction for incompressible flows using deformable hybrid grids," Journal of Computational Physics, vol. 143, no. 2, pp. 569597, 1998.

[52] K. Raghavan and M. M. Bernitsas, "Experimental investigation of reynolds number effect on vortex induced vibration of rigid circular cylinder on elastic supports," Ocean Engineering, vol. 38, no. 5-6, pp. 719-731, 2011. 


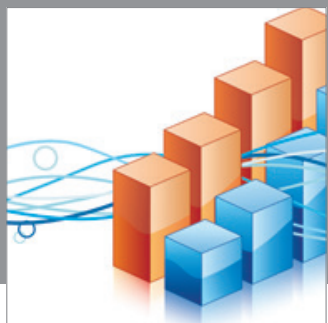

Advances in

Operations Research

mansans

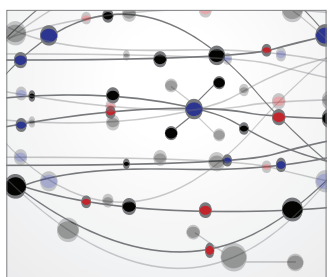

The Scientific World Journal
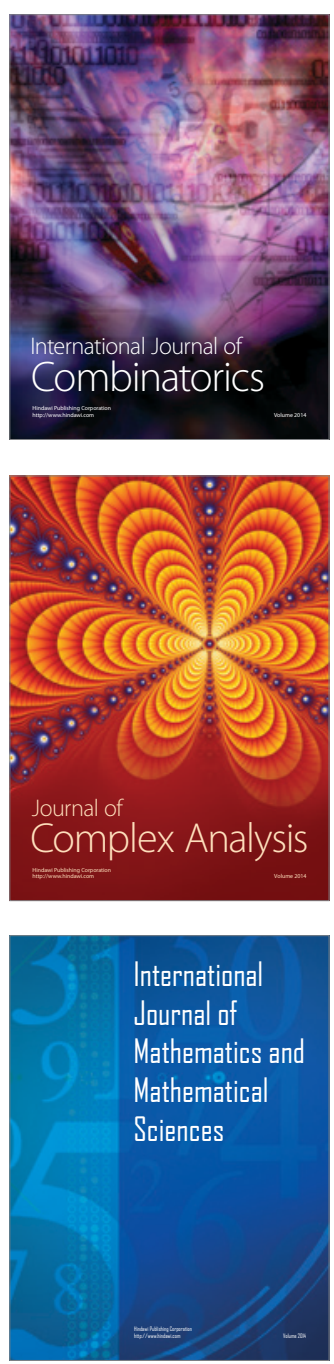
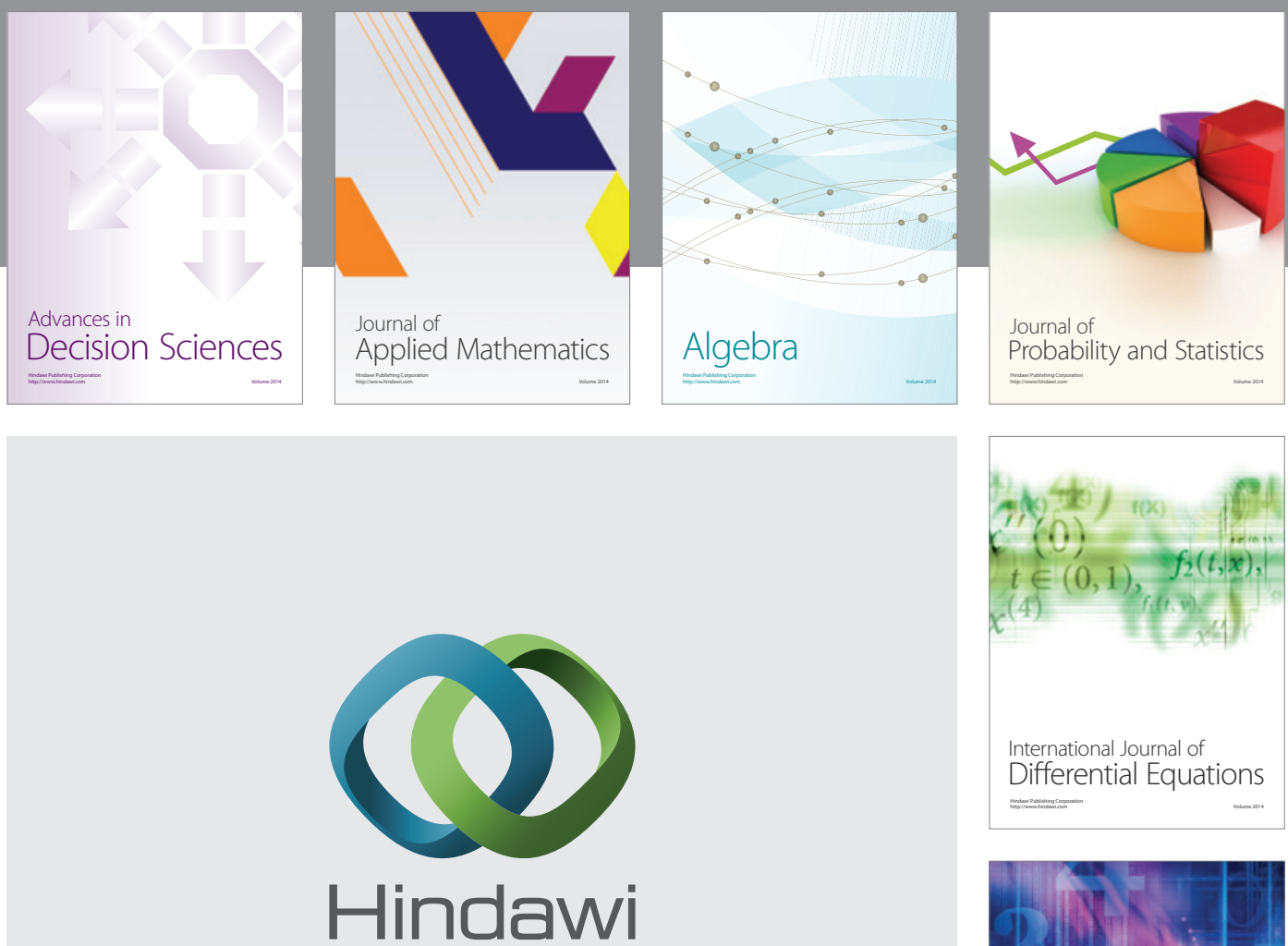

Submit your manuscripts at http://www.hindawi.com
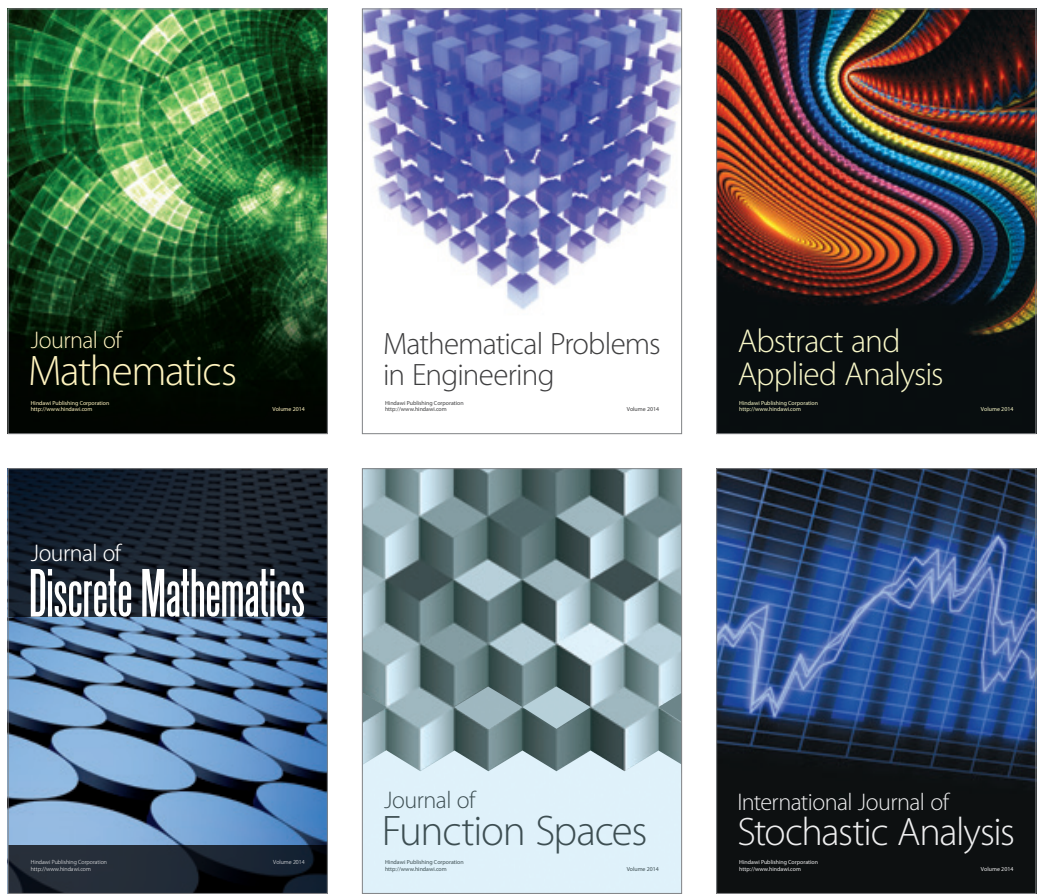

Journal of

Function Spaces

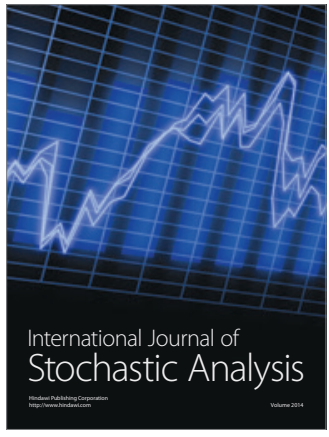

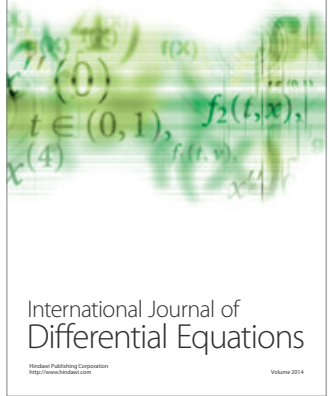
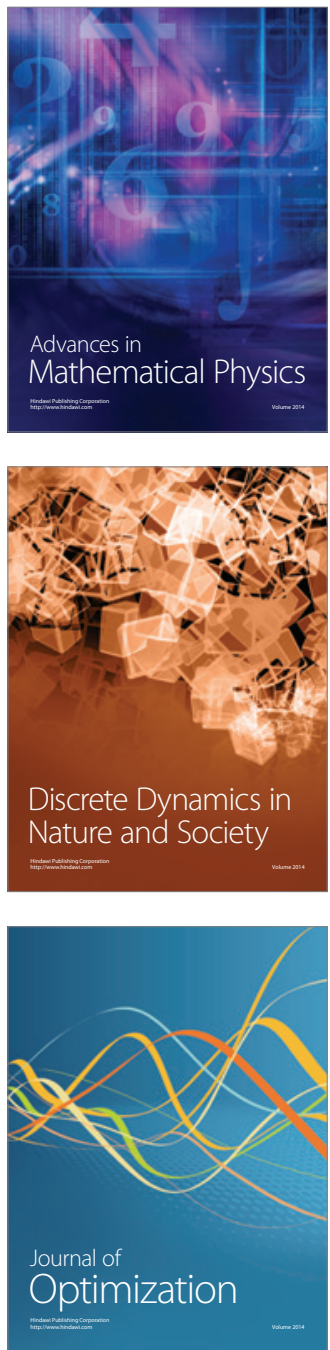